\title{
LIFESPAN DISTRIBUTION OF SIMD GROUPS ON A GPU ENGAGED IN A CLASS OF PROBABILISTIC COMPUTATION
}

\author{
Masanari Iida* and Naoto $\mathrm{Niki}^{\dagger}$
}

\begin{abstract}
In each SIMD (Single Instruction, Multiple Data) group, called a 'warp' of a GPU (Graphics Processing Unit), all the fixed number of threads execute the same instruction concurrently at each unit period of time. We consider a class of probabilistic algorithms designed for use on GPUs, including a wide variety of Monte Carlo methods, such that each thread contains a loop iterated stochastically variable times, and that the life-cycle of a warp ends when the slowest thread completes its requested task. A run-time model is proposed in order to explain the distributions of execution time observed in SIMD parallel computations using the algorithms of this class. Asymptotic properties of those distributions are also presented.
\end{abstract}

\section{Introduction}

Most of modern high-grade GPUs (Graphics Processing Units) have hundreds or thousands of cores (independent processing units) on their boards, which realize remarkably high performance in image processing by using SIMD (Single Instruction, Multiple Data) parallel technologies. Their use in a wide variety of computation other than 2D or 3D graphics, including weather dynamics and gas-liquid flow analysis, is known as GPGPU (GeneralPurpose use of GPU) and attracts attention of many workers engaged in various fields of science and technology (see, e.g., Shimokawabe et al., 2011).

In GPUs of SIMD architecture, each thread belongs to a SIMD group, comprised of a unit number (denoted by $m$, typically $m=32$ or 64 ) of threads and called a 'warp' for those designed by nVIDIA, where a single common instruction is executed in each cycle of time (nVIDIA Corp., 2009). When a conditional branching happens, every thread in the warp may execute both if-then and else branches, but those threads which must not follow a given branch perform no-operations (denoted by nops) instead without any consequence. This probable inefficiency has induced workers to avoid implementing probabilistic algorithms on GPUs (Lee et al., 2010, for instance), since they essentially involve many chances of conditional branching, despite the fact that GPGPU might be promising in a wide range of Monte Carlo computations.

On the other hand, in our preliminary experiments (Iida and Niki, 2013), we have observed that the more pseudo-random variates we generate by using rejection sampling in a thread on a GPU, the less run-time per variate we have. This fact stimulates us to design a run-time model discussed in this article for a particular class of probabilistic algorithms, including random walks and Monte Carlo methods with importance sampling, in each of

\footnotetext{
*Graduate School of Enginerring, Tokyo University of Science E-mail: masanari@ms.kagu.tus.ac.jp

${ }^{\dagger}$ Department of Management Science, Tokyo University of Science E-mail: niki@ms.kagu.tus.ac.jp Key words: GPGPU; Monte Calro computation; Parallel computing; Probabilistic algorithm; Random number generation; Rejection sampling
} 
which a block of instructions (called a 'trial' here) is repeated a randomly varying number of times.

The main target of this article is to give an approximation to the distribution of execution time observed in SIMD parallel configuration of a large number of cores. The results might be also useful in the design of GPGPU algorithms with stochastic behavior, because of the theoretically endorsed fact that well-organized and simplified probabilistic algorithms may be several tens of times or more, more efficient than those on a fast CPU in spite of existing negative reports.

\section{Implementing Probabilistic Algorithms on GPU}

\subsection{A brief introduction to GPGPU}

There are two principal merits in GPGPU. One is its superior parallel computing performance: a GPU consists of a great number of efficient cores designed for parallel performance, while a CPU consists of a few cores. Hardware implementation of elementary functions, e.g. logarithmic and trigonometric functions, in Super Function Units (SFUs) also attracts us especially in statistical computing. The other is its superb cost performance: GPUs are much cheaper than PC systems and can be equipped simply by inserting them in fast PCI Express buses built into almost all the latest motherboards for desktop PCs.

Two brands of GPUs are used worldwide, one is nVIDIA and the other is AMD (AMD, 2012). We focus only upon nVIDIA GPUs and use nVIDIA terminology, throughout this article, but the discussion here can also be applied to products by AMD and other vendors.

nVIDIA has been providing a program development environment called CUDA with which we can compose programs not only for multiple SIMD processes on a GPU, but also for bidirectional communication between CPU and GPU.

The GPU part of a job, called a 'grid', is started by a CPU request after its compiled codes have been sent through the fast PCI bus lines. A grid has exclusive use of a requisite number of GPU blocks called 'streaming multiprocessors', each of which provides computing resources, including cores, registers, function units, local and shared memories, to a 'block' of threads. If two or more blocks are allocated to a multiprocessor, they are consecutively processed.

Each 'block' consists of several 'warps', i.e., SIMD groups of threads. The number of warps in a block is chosen to produce the optimum throughput depending the GPU architecture and the job. See 'Cuda C programming guide' (nVIDIA Corp., 2013) for more details.

\subsection{Use of GPU in statistical computing including Monte Carlo calculation}

It seems necessary to describe some details of the inefficiency commonly found in SIMD architecture mentioned in the introduction section.

Consider a simple case with two if-then-else blocks shown in Figure 1. If the logical variable $P($ e.g., $P=(\mathrm{PARAM}>0))$ has the same value (true in this example) for all the $m$ threads of a warp, then each thread executes only one branch (i.e., B, the if-then branch). On the contrary, when there exist one true and one false, at least, among the truth values of $Q(e . g ., Q=(\operatorname{rnd}() \leq 0.5))$ in the warp, then each thread executes both branches (in the optimal fixed order of execution; either $D$ before $E$, or, $E$ before $D$ ), provided that those threads which do not logically follow a given branch are flagged and make no effective action (shown as nop).

This architecture may involve severe inefficiency in speed, if the latter case with mixed 


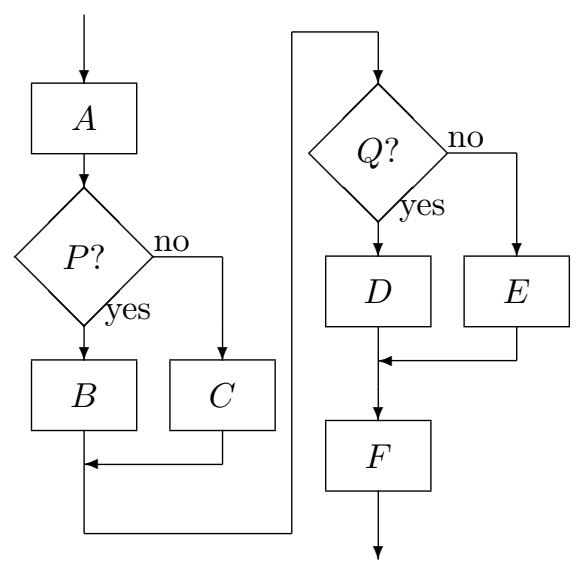

\begin{tabular}{cccccc}
\multicolumn{8}{c}{ thread ID } \\
0 & 1 & 2 & 3 & $\cdots$ & $m-1$ \\
\hline$\vdots$ & $\vdots$ & $\vdots$ & $\vdots$ & $\cdots$ & $\vdots$ \\
$A$ & $A$ & $A$ & $A$ & $\cdots$ & $A$ \\
$P:$ true & true & true & true & $\cdots$ & true \\
$B$ & $B$ & $B$ & $B$ & $\cdots$ & $B$ \\
$Q:$ true false & false & true & $\cdots$ & true \\
$D$ & nop & nop & $D$ & $\cdots$ & $D$ \\
nop & $E$ & $E$ & nop & $\cdots$ & nop \\
$F$ & $F$ & $F$ & $F$ & $\cdots$ & $F$ \\
$\vdots$ & $\vdots$ & $\vdots$ & $\vdots$ & $\cdots$ & $\vdots$ \\
\hline
\end{tabular}

Fig. 1: SIMD operations in a warp with conditional branches.

truth values happens frequently, providing either $D$ or $E$ (or both) comprises a time consuming procedure. However, changing our viewpoints and considering the fact that no pipeline stall due to the branch takes place between $B$ and $F$, we can rewrite the same preceding sentence as follows: "If both if-then and else branches consist of only a few operations, the overhead of conditional divergence is acceptable, even if the divergence quite frequently happens."

The above circumstances appear in various kinds of statistical computations. The Metropolis method for numerical evaluation of the exact $p$-value for a given contingency table under the null hypothesis of independence, for example, involves full conditional transitions from a table to one of its neighboring tables. Monte Carlo composition of the sampling distribution of a statistic is another typical example, where a large number of samples from a population are generated for making a sequence of pseudo observations of a statistic. If rejection and/or composition techniques are applied in generation of random numbers distributed with a specified distribution one or more conditional branches take place for each random variate.

\section{Run-time Model for SIMD Computation}

\subsection{Probabilistic algorithms with repetition of an identical procedure}

We engage in discussions, in this article, on a class of probabilistic algorithms, including a wide variety of Monte Carlo methods. Each algorithm in this class consists a huge number of repeated execution of a probabilistic procedure which contains a stochastic number of trials. We first propose a run-time model for this class.

Consider a grid, the GPU part of a job, of which the goal is to obtain a huge number $k \times \ell$ of pseudo observations for a random variable. Parallel computation is performed by equally distributing the requisite procedures into $\ell$ threads. As shown in Figure 2, each thread belongs to a warp, the SIMD group, together with $m-1$ other threads and is required to obtain $k$ independent observations.

If the number of trials varies for each observation, we need a special structure in designing the algorithm. For example, the flowchart (a) in Figure 3 is designed in order to follow the SIMD law in a faithful manner, where we can use a 'vote' family command for checking the allover status of threads in the warp. On the other hand, at the first glance, the 


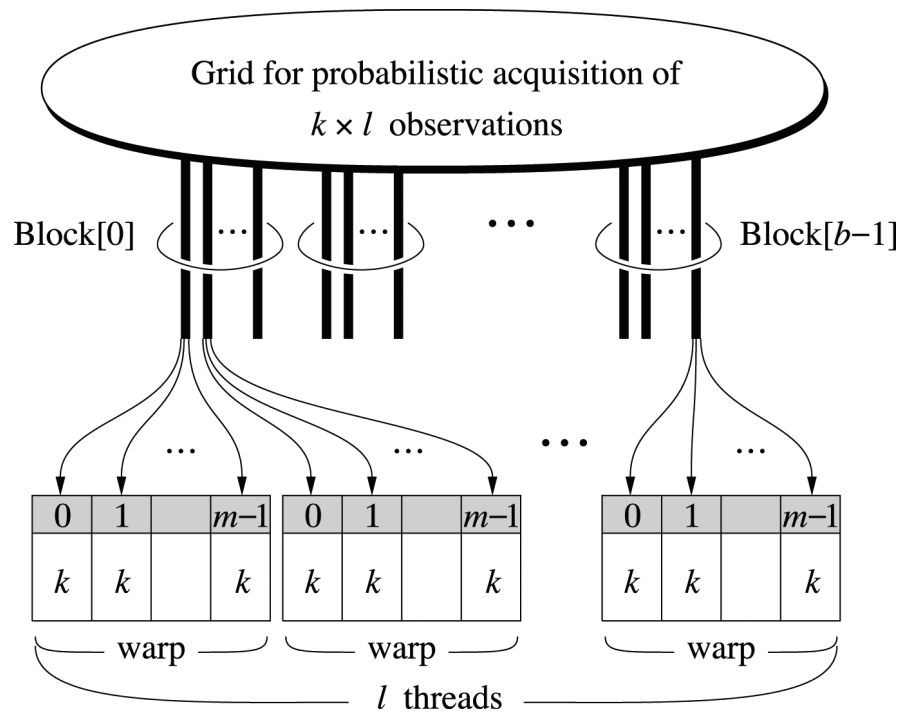

Fig. 2: Distributing procedures into threads of a grid

(a) satisfies SIMD law without effectual overhead.

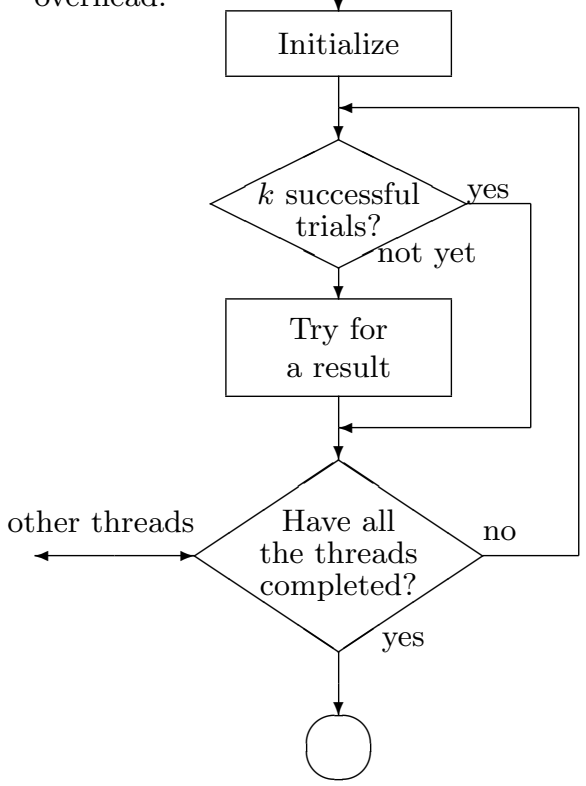

(b) seems to violate SIMD law but works in some GPUs.

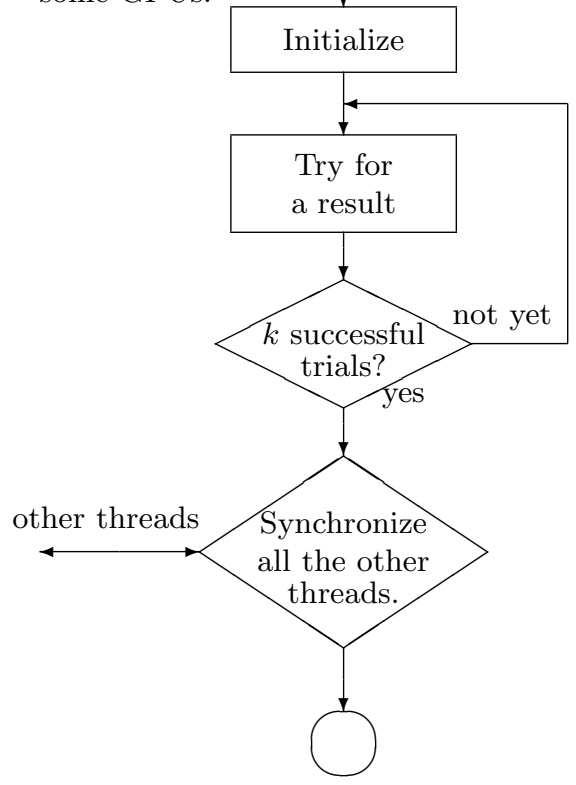

Fig. 3: SIMD flow of each thread in a warp. 


\begin{tabular}{ccccccccc}
\multicolumn{10}{c}{ threads } \\
trial & 0 & 1 & 2 & 3 & 4 & $\cdots$ & $m-1$ & \\
\hline 1 & - & $\circ$ & $\circ$ & - & $\circ$ & $\cdots$ & - & \\
2 & $\circ$ & $\circ$ & - & $\circ$ & $\circ$ & $\cdots$ & $\circ$ & $-:$ succeeded \\
3 & - & $\bullet$ & $\circ$ & $\circ$ & - & $\cdots$ & $\circ$ & $\bullet:$ dailed \\
4 & - & $\bullet$ & $\bullet$ & - & $\bullet$ & $\cdots$ & $\bullet$ & \\
5 & $\circ$ & $\bullet$ & $\bullet$ & $\bullet$ & $\bullet$ & $\cdots$ & $\bullet$ & \\
6 & $\bullet$ & $\bullet$ & $\bullet$ & $\bullet$ & $\bullet$ & $\cdots$ & $\bullet$ &
\end{tabular}

Fig. 4: Example of probabilistic SIMD computation in a warp for $k=3$.

flowchart (b) seems to violate the SIMD law, but works correctly on some (possibly many) GPUs. Here, synchronization can be done either explicitly by a system call in the program or implicitly by the warp management system.

Figure 4 shows an example of status changes in each thread of a warp during its lifespan. The fastest thread has completed its role at the third trial, in this example. In contrast, the most unlucky thread has needed 6 trials.

\subsection{Distribution of the number of trials within a warp}

For given positive integers $m$ and $k$, let $\left(X_{n t}\right)_{t \in \mathcal{K}}$ be a truncated recurrent renewal chain which represents how many trials have been done to obtain the $t$-th result in the $n$-th thread of a warp, where $\mathcal{K}=\{0,1, \ldots, k\}$ and $n \in \mathcal{M}=\{1,2, \ldots, m\}$. In the other words, the waiting times in $\left\{X_{n t}-X_{n, t-1} \mid n \in \mathcal{M}, t \in \mathcal{K}-\{0\}\right\}$ are i.i.d. positive integers, provided $X_{n 0}=0$ and $X_{n k}<\infty$, each of which signifies the number of trials until a success happens. Let $Y$ and $Z$ be the random variables

$$
Y=\max _{n \in \mathcal{M}} X_{n k}, \quad Z=\min _{n \in \mathcal{M}} X_{n k} .
$$

then the number of trials in a warp is given by $Y$ as described in the previous subsection.

Let $F_{k}(x)$ and $f_{k}(x)$ be the cumulative distribution function $(c d f)$ and the probability mass function (pmf) of $X_{n k}$, respectively, providing $F_{k}(x)=f_{k}(x)=0$ for any $x \leq 0$. Then, the $c d f H_{m}^{(k)}(x)$ and the $p m f h_{m}^{(k)}(x)$ of $Y$ are given as

$$
H_{m}^{(k)}(x)=F_{k}(x)^{m}, \quad h_{m}^{(k)}(x)=F_{k}(x)^{m}-F_{k}(x-1)^{m} .
$$

In any practical parallel algorithm, except experimental ones, we can assume the condition below:

Assumption 1 All the moments of $F_{1}$ are finite.

Either with or without Assumption 1, the following lemma is straightforward.

Lemma 1 Let $\psi_{k}(s)$ and $\psi_{1}(s)$ be the characteristic functions for $F_{k}$ and $F_{1}$, respectively, then $\log \psi_{k}(s)=k \log \psi_{1}(s)$.

From this lemma under Assumption 1, noting $\log \psi_{1}(s)$ and $\log \psi_{k}(s)$ are the cumulant generating functions, we have several corollaries.

Corollary $1 \mathrm{E} X_{n k}=k \mu$ and $\operatorname{Var} X_{n k}=k \sigma^{2}$, where $\mu=\mathrm{E} X_{n 1}$ and $\sigma^{2}=\operatorname{Var} X_{n 1}$.

Corollary 2 The $j$-th cumulant $\kappa_{j}^{*}$ for the standardized variable $\left(X_{n k}-k \mu\right) / \sqrt{k \sigma^{2}}$, satisfies the so-called Cornish-Fisher assumption, i.e., $\kappa_{1}^{*} \sim O\left(k^{-\frac{1}{2}}\right)$ and $\kappa_{j}^{*} \sim O\left(k^{\frac{2-j}{2}}\right)$ for $j \geq 2$. 
Corollary $3 X_{n k}$ has the limiting normal distribution $N\left(k \mu, k \sigma^{2}\right)$ as $k \rightarrow \infty$.

\subsection{The repeat count $k$ should be large enough}

It might be most probable that one would implement the probabilistic algorithm shown in Figure 3 with $k=1$ and repeat it $k$ times considering the SIMD architecture of GPUs. In those cases, the number of trials $Y^{\prime}$ of each thread in a warp is given as

$$
Y^{\prime}=\sum_{i=1}^{k} \max _{n \in \mathcal{M}} X_{n 1}^{(i)}, \quad X_{n 1}^{(i)} \stackrel{i . i . d .}{\sim} F_{1},
$$

where nops are frequently executed in vain only to wait for the slowest thread in each iteration.

The expected value $\mathrm{E} Y^{\prime}$ is usually much larger than $\mathrm{E} Y$. For instance, consider the case that $F_{1}$ is the geometric distribution with probability parameter $p$, then

$$
\begin{aligned}
\mathrm{E} Y^{\prime} & =k \sum_{x=1}^{\infty} x\left(\left(1-(1-p)^{x}\right)^{m}-\left(1-(1-p)^{x-1}\right)^{m}\right) \\
& \approx 3.428 k \quad\left(\text { for } m=32, p=\frac{3}{4}\right),
\end{aligned}
$$

On the other hand, by using the $c d f$ of a negative binomial distribution,

$$
\begin{aligned}
\mathrm{E} Y & =\sum_{x=1}^{\infty} x\left(I_{p}(k, x-k+1)^{m}-I_{p}(k, x-k)^{m}\right) \\
& \approx \frac{4 k \sqrt{k}+5.984 k+4.845 \sqrt{k}-0.612}{3 \sqrt{k}+1.383}+\frac{0.283}{\sqrt{k+1}+0.121} \\
& \quad\left(\text { for } m=32, p=\frac{3}{4}\right),
\end{aligned}
$$

where $I_{p}(a, b)$ signifies the regularized incomplete beta function $B(p ; a, b) / B(a, b)$. The above facts imply that a crude parallel algorithm corresponding to $Y^{\prime}$ will do, on average, more than 2 or 2.5 times more trials than the algorithm shown in Figure 3 for $k \geq 18$ or $k \geq 1,400$, respectively, in this example.

One may have noticed that the title of this subsection is demonstrated in (5) in an explicit form. Table 1 shows the expected numbers of trials for obtaining 10,000 results. From this table, it can be said that $k$ should be tens or larger as far as total efficiency of computation attains its maximum with optimized resource allocation.

Table 1: Average numbers of trials for 10,000 results.

\begin{tabular}{rrrrl}
$k$ & $\mathrm{E} Y$ & $k^{\prime}=10^{4} / k$ & $k^{\prime} \mathrm{E} Y$ & \\
\cline { 1 - 4 } 1 & 3.4279 & 10,000 & 34,279 & \\
2 & 5.4000 & 5,000 & 27,000 & \\
5 & 10.598 & 2,000 & 21,196 & \\
10 & 18.577 & 1,000 & 18,577 & \\
20 & 33.744 & 500 & 16,872 & \\
50 & 77.354 & 200 & 15,471 & \\
100 & 148.08 & 100 & 14,808 & \\
500 & 698.48 & 20 & 13,970 & \\
1,000 & $* 1,377.9$ & 10 & $* 13,779$ & $*$ \\
10,000 & $* 13,472$ & 1 & $* 13,472$ & extrapolated values \\
\cline { 1 - 3 } & & & &
\end{tabular}




\subsection{The limiting distribution of $Y$ and its approximation}

Hereafter, we discuss the cases where $k$ is large enough (usually twenty or more under Assumption 1, say) to make the normal approximation in Corollary 3 practical. In the following discussions, Assumption 1 can be weakened as far as the central limit theorem holds for the distribution of $X_{n k}$ for $k \rightarrow \infty$.

Let $\Phi(x)$ and $\phi(x)$ be the $c d f$ and $p d f$ of the standard normal distribution, respectively. Then, the limiting distribution of $(Y-k \mu) / \sqrt{k \sigma^{2}}$ for $k \rightarrow \infty$ has the $c d f H_{m}(x)=H_{m}^{(\infty)}(x)=$ $\Phi(x)^{m}$ and the $p d f h_{m}(x)=m \phi(x) \Phi(x)^{m-1}$. See, e.g., Gumbel (1947).

It is well known that $H_{m}$ tends to a Gumbel distribution as $m \rightarrow \infty$, but the speed of convergence is too slow to use this fact for numerical computation in the typical cases, i.e., $m=32$ or 64 . Alternatively, we have found that $H_{32}$ can be closely approximated by using a three parameter gamma distribution, $\operatorname{Gamma}(13, \sqrt{\pi} / 13, \sqrt{\pi} / 6)$, where the parameters are chosen for mnemonic sake and can be replaced with optimal ones only a little superior in accuracy. Actually, for any $x \geq \sqrt{\pi} / 6$,

$$
\left|h_{32}(x)-\frac{13^{13}}{12 ! \pi^{13 / 2}}\left(x-\frac{\sqrt{\pi}}{6}\right)^{12} \exp \left(-\frac{13}{\sqrt{\pi}}\left(x-\frac{\sqrt{\pi}}{6}\right)\right)\right|<0.02,
$$

and the first six cumulants $\nu_{j}(j=1, \ldots, 6)$ of $H_{32}$ are also approximated with differences less than 0.006:

$$
\begin{aligned}
& \nu_{1}-\frac{7 \sqrt{\pi}}{6} \approx 0.002, \quad \nu_{2}-\frac{\pi}{13} \approx-0.00004, \quad \nu_{3}-\frac{2 \pi^{3 / 2}}{13^{2}} \approx-0.0002, \\
& \nu_{4}-\frac{3 ! \pi^{2}}{13^{3}} \approx 0.006, \quad \nu_{5}-\frac{4 ! \pi^{5 / 2}}{13^{4}} \approx 0.004, \quad \nu_{6}-\frac{5 ! \pi^{3}}{13^{5}} \approx-0.0006 .
\end{aligned}
$$

Similar approximation can be also applicable to $H_{m}$ for a wide range of $m$.

\subsection{Distribution of run-time for a trial}

Let $R_{j}$ denote the run-time of the $j$-th trial, which is common to every thread of a warp because of SIMD operation. In the probabilistic algorithms concerned, $R_{j}$ can be considered as a discrete random variable distributed with a distribution $G_{c}$, where $c$ is the number of active threads in the warp at the $j$-th trial, i.e.,

$$
c=c(j)=\#\left\{n \in \mathcal{M} \mid X_{n k} \geq j\right\} .
$$

Unless the programming code contains some kind of bug, it can be said, as seen in Figure 5, that

1. The support of $G_{c}$ comprises several fixed points of time corresponding to all the possible paths through the conditional branches in a trial and to all the possible delays caused by memory access fluctuations.

2. $G_{c}$ is almost a one point distribution when $c$ is large enough, like $G_{32}$ in Figure 5.

3. As $c$ decreases from $m$ to 1 , the possibility of taking the slowest path through all the if-then and else blocks becomes smaller.

4. As a result of the above, the expectation of $R_{j}$ decreases as $j$ increases beyond $Z$. 

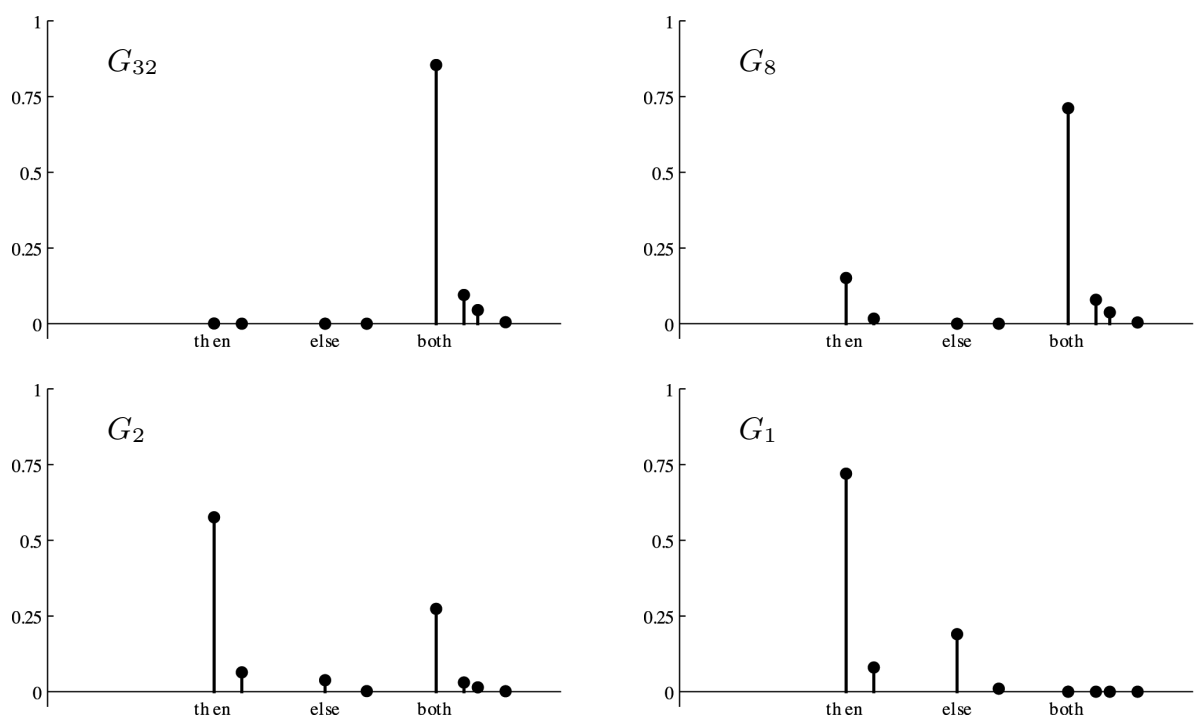

Fig. 5: An example of $\left\{G_{m}, \ldots, G_{1}\right\}(m=32)$ with a single conditional branch.

From these, the following assumption is posited hereafter.

Assumption 2 For any $c \in \mathcal{M}$, the support of $G_{c}$ is included within a fixed interval $[a, b]$ and, as a consequence, all the $r$-th cumulants $\rho_{c r}(r=1,2 \ldots)$ of $G_{c}$ are finite; providing the sequence of means is descending as c runs from $m$ to 1 , i.e.,

$$
\rho_{m 1} \geq \rho_{(m-1) 1} \geq \cdots \geq \rho_{21} \geq \rho_{11} .
$$

The relative relations in (9) would hold for carefully designed algorithms. Even if some inequlities may not hold, there exists a clearly declining tendency.

\section{Approximation for Lifespan Distribution of a Warp}

\subsection{Lifespan time $L_{Y}$ of a warp}

Providing a random variable $R_{0}$ with finite cumulants to represent the total time spent for preceding and succeeding operations to the part of iterating trials, we can write the lifetime $L_{Y}$ of a warp as

$$
L_{Y}=\sum_{j=0}^{Y} R_{j}=L_{Z}+\sum_{j=Z+1}^{Y} R_{j}, \quad L_{Z}=R_{0}+\sum_{j=1}^{Z} R_{j} .
$$

where each of $\left\{R_{j} \mid j=1, \ldots, Z\right\}$ is independently distributed with $G_{m}$.

Concerning the distributions of $L_{Y}$, the following lemma plays an essential role.

Lemma $2 \quad$ As $k \rightarrow \infty, Y / k \stackrel{P}{\rightarrow} \mu$ and $(Y-Z) / k \stackrel{P}{\rightarrow} 0$.

Proof. From Corollary 1, for any $d>0$, the Chebyshev inequality gives

$$
\begin{aligned}
\operatorname{Pr}\{Y-k \mu \geq d\} & =\operatorname{Pr}\left\{\left(X_{1 k}-k \mu \geq d\right) \vee \cdots \vee\left(X_{m k}-k \mu \geq d\right)\right\} \\
& \leq \operatorname{Pr}\left\{X_{1 k}-k \mu \geq d\right\}+\cdots+\operatorname{Pr}\left\{X_{m k}-k \mu \geq d\right\} \\
& \leq m \operatorname{Pr}\left\{\left|X_{1 k}-k \mu\right| \geq d\right\} \leq \frac{m k \sigma^{2}}{d^{2}},
\end{aligned}
$$


and, in similar manner,

$$
\operatorname{Pr}\{k \mu-Z \geq d\} \leq \frac{m k \sigma^{2}}{d^{2}}
$$

Hence, for any $\varepsilon>0, \delta>0$ and $k>\frac{8 m \sigma^{2}}{\delta \varepsilon^{2}}$, it holds that

$$
\begin{aligned}
\operatorname{Pr}\left\{\frac{Y-Z}{k}>\varepsilon\right\} & =\operatorname{Pr}\{(Y-k \mu)+(k \mu-Z)>\varepsilon k\} \\
& \leq \operatorname{Pr}\left\{\left(Y-k \mu>\frac{\varepsilon k}{2}\right) \vee\left(k \mu-Z>\frac{\varepsilon k}{2}\right)\right\} \\
& \leq \operatorname{Pr}\left\{Y-k \mu>\frac{\varepsilon k}{2}\right\}+\operatorname{Pr}\left\{k \mu-Z>\frac{\varepsilon k}{2}\right\} \leq \frac{8 m \sigma^{2}}{k \varepsilon^{2}}<\delta .
\end{aligned}
$$

and also

$$
\begin{aligned}
\operatorname{Pr}\left\{\left|\frac{Y}{k}-\mu\right|>\varepsilon\right\} & =\operatorname{Pr}\{Y-k \mu>\varepsilon k\}+\operatorname{Pr}\{k \mu-Y>\varepsilon k\} \\
& \leq \operatorname{Pr}\{Y-k \mu>\varepsilon k\}+\operatorname{Pr}\{k \mu-Z>\varepsilon k\} \leq \frac{2 m \sigma^{2}}{k \varepsilon^{2}}<\delta .
\end{aligned}
$$

Lemma 3 Assume that $R_{0}$ has a finite upper bound, then, as $k \rightarrow \infty$,

$$
\frac{L_{Y}}{k} \stackrel{P}{\rightarrow} \frac{L_{Y}^{\prime}}{k}=\frac{1}{k} \sum_{j=1}^{Y} R_{j}^{\prime}, \quad R_{j}^{\prime} \stackrel{\text { i.i.d. }}{\sim} G_{m}
$$

Proof. For $1 \leq j \leq Z$, noting $R_{j}^{i . i . d}{ }^{\sim} G_{m}$, we can replace $R_{j}^{\prime}$ with $R_{j}$. Hence, by noting Assumption 2, Lemma 2 gives

$$
\begin{aligned}
\left|\frac{L_{Y}-L_{Y}^{\prime}}{k}\right| & \leq \frac{1}{k}\left(R_{0}+\sum_{j=Z+1}^{Y}\left|R_{j}-R_{j}^{\prime}\right|\right) \\
& \leq \frac{\bar{R}}{k}+\frac{Y-Z}{k}(b-a) \stackrel{P}{\rightarrow} 0 \quad(k \rightarrow \infty),
\end{aligned}
$$

where $\bar{R}$ is the upper bound of $R_{0}$.

Usually, the difference $\left|R_{j}-R_{j}^{\prime}\right|$ in (15) is small even in the case that $R_{j} \sim G_{1}$. Then, $L_{Y}^{\prime}$ gives an approximation to $L_{Y}$ precise enough to use in practical applications.

\subsection{The limiting distribution of $L_{Y}^{\prime} / k$}

The cumulants of the limiting distribution of $L_{Y}^{\prime} / k$ as $k \rightarrow \infty$ are directly obtained from Lemmas 1 and 3 of this article and Theorem 1 in Niki et al. (1990).

Theorem 1 Let $\nu_{s}$ and $\rho_{s}$ denote the $s$-th cumulant of $H_{m}$ and $G_{m}$. Then, the $s$-th cumulant $\lambda_{s}$ of the limiting distribution of $L_{Y}^{\prime} / k$ is given as

$$
\lambda_{s}=\sum_{\pi(s)} \frac{s !}{r_{1} ! r_{2} ! \cdots r_{s} !(1 !)^{r_{1}}(2 !)^{r_{2}} \cdots(s !)^{r_{s}}} \nu_{q} \rho_{1}^{r_{1}} \rho_{2}^{r_{2}} \cdots \rho_{s}^{r_{s}},
$$

where the summation goes over all partitions $\pi(s)$ of $s$, i.e., 


$$
\pi(s)=\left\{\left(1^{r_{1}} 2^{r_{2}} \cdots s^{r_{s}}\right) \mid\left(r_{1}, r_{2}, \ldots, r_{s}\right) \in \mathbb{N}^{s}, r_{1}+2 r_{2}+\cdots+s r_{s}=s\right\}
$$

with $q=r_{1}+r_{2}+\cdots+r_{s}$.

Corollary 4 The first four cumulants are given as follows:

$$
\begin{aligned}
& \lambda_{1}=\nu_{1} \rho_{1}, \quad \lambda_{2}=\nu_{2} \rho_{1}^{2}+\nu_{1} \rho_{2}, \quad \lambda_{3}=\nu_{3} \rho_{1}^{3}+3 \nu_{2} \rho_{1} \rho_{2}+\nu_{1} \rho_{3}, \\
& \lambda_{4}=\nu_{4} \rho_{1}^{4}+6 \nu_{3} \rho_{1}^{2} \rho_{2}+\nu_{2}\left(4 \rho_{1} \rho_{3}+3 \rho_{2}^{2}\right)+\nu_{1} \rho_{4} .
\end{aligned}
$$

Corollary 5 If $G_{m}$ can be approximated with a one point distribution, like $G_{32}$ in Figure 5 , then, since $\rho_{j} \approx 0(j \geq 2)$,

$$
\lambda_{1} \approx \nu_{1} \rho_{1}, \quad \lambda_{2} \approx \nu_{2} \rho_{1}^{2}, \quad \lambda_{3} \approx \nu_{3} \rho_{1}^{3}, \quad \lambda_{4} \approx \nu_{4} \rho_{1}^{4}, \quad \cdots,
$$

which mean that $L_{Y}^{\prime} / k$ can be approximated with a gamma distribution.

Remark 1 Similar discussion gives that the distribution of $L_{Z} / k$ can be approximated with a 'reversed' gamma distribution of which the $p d f$ is the reflected image of a 'usual' gamma density. Correctness of approximation would be more precise than that for $L_{Y} / k$.

Remark 2 From the inequalities in (9), there exists a clear tendency that, for the larger value of $Y$, the difference $R_{j}^{\prime}-R_{j}$ has the larger expected value. This fact implies that the scale and location parameters of the best-fit gamma distribution for $L_{Y} / k$ are a little smaller than those for $L_{Y}^{\prime} / k$. On the other hand, the shape parameter for $L_{Y} / k$ is larger than that for $L_{Y}^{\prime} / k$ because of smaller skewness.

\section{Monte Calro Computation with Envelope Rejection — an Example}

\subsection{Random number generation with envelope rejection}

Consider a weighted Monte Calro integration of a designated function $w(x)$, i.e.,

$$
\int_{-\infty}^{\infty} w(x) v(x) d x \approx \frac{1}{N} \sum_{j=1}^{N} w\left(V_{j}\right)
$$

where $V_{1}, \ldots, V_{N}$ are random variates independently distributed with a $p d f v(x)$. It is well known that the accuracy of approximation is proportional to $1 / \sqrt{N}$.

Envelope rejection is one of the most popular techniques for generating a random variate $V \sim v(x)$ and is applicable if there exists a $p d f q(x)$ such that $(\forall x, 0<\exists p<1) p v(x) \leq q(x)$ and the random variate $Q \sim q(x)$ is generated fast. An outline of the generation procedure can be written as follows:

1. Generate a pair of random variates $(Q, U)$ where $U \sim \operatorname{Uniform}[0,1]$.

2. If $U q(x) \leq p v(x)$ accept $Q$ as $V$, otherwise go back to (1).

The probability of acceptance is $p$.

When we use $\ell=N / k$ threads, i.e., $\ell / m=N /(k m)$ warps on a GPU for this numerical integration, the number $X$ of trials made in a thread for generating $k$ random variates has the negative binomial distribution with the $p m f$

$$
f_{k}(x)=\left(\begin{array}{l}
x-1 \\
k-1
\end{array}\right) p^{k}(1-p)^{x-k} \quad(x \geq k)
$$


of which first four cumulants are given, reflecting Lemma 1, as

$$
\kappa_{1}=\frac{k}{p}, \quad \kappa_{2}=\frac{k(1-p)}{p^{2}}, \quad \kappa_{3}=\frac{k(1-p)(2-p)}{p^{3}}, \quad \kappa_{4}=\frac{k(1-p)\left(p^{2}-6 p+6\right)}{p^{4}} .
$$

For numerical illustration, the method due to Ahrens and Dieter (1974) is used here for generating gamma variates with shape parameter $1 / 2$, i.e.,

$$
v(x)=\frac{1}{\sqrt{\pi}} x^{-\frac{1}{2}} e^{-x}, \quad q(x)=\left\{\begin{array}{l}
\frac{p}{\sqrt{\pi}} x^{-\frac{1}{2}} \text { for } x \leq 1 \text { with probability } p_{0}, \\
\frac{p}{\sqrt{\pi}} e^{-x} \text { for } x>1 \text { with probability } 1-p_{0},
\end{array}\right.
$$

where

$$
\mu=\frac{1}{p}=\frac{e \sqrt{\pi}}{1+2 e} \approx 1.336, \quad p_{0}=\operatorname{erf}(1) \approx 0.843 .
$$

Then, a trial has three conditional branches with $3^{3}=27$ possible execution paths, which makes the difference $\left|R_{j}^{\prime}-R_{j}\right|(j>Z)$ not negligible.

\subsection{Gamma distributions fitted to empirical lifespan distributions}

An empirical distribution of $L_{Y} / k$ observed in the Monte Calro integration of $w(x)=x^{2}$ with $V \sim v(x)$ in (22) is shown as the histogram with gray bars in Figure 6. The integration has been done on an nVIDIA Tesla C2070 with 448 cores and clock rate of $1.147 \mathrm{GHz}$, by letting $N=448 \times 1,000$ and $k=1,000$. The solid line represents the $p d f$ of the fitted gamma distribution Gamma(21, 0.00893, 6.915) to the observations.

The dashed line represents the imaginary distribution of $L_{Y}^{\prime} / k$ drawn as the $p d f$ of Gamma(13, 0.01363, 6.939). These parameters are brought from the best-fit distribution ReversedGamma $(13,0.01363,6.880)$ to the empirical distribution of $L_{Z} / k$, which is expressed as the histogram with white bars. As stated in Remark 1, the location parameter is given by using $6.939 \approx 6.880+2 \times 0.01363 \times 13 / 6$. In addition, as noted in Remark 2 , the distribution of $L_{Y} / k$ has a larger shape parameter and smaller scale and location parameters than those for $L_{Y}^{\prime} / k$, correspondingly.

All the CUDA codes in C++, both those run on GPU and on CPU together, are compiled and executed through CUDALink, the CUDA interface built in Mathematica (see Wolfram Research Inc., 2013, for details).

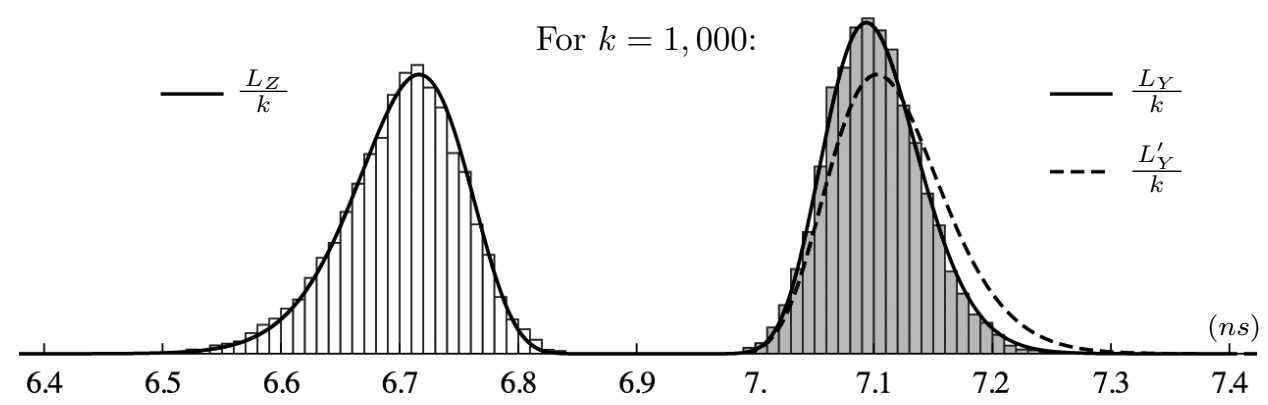

Fig. 6: Lifespan distribution of warps in gamma random number generation 


\section{Concluding Remarks}

Not only in Monte Calro integration but in various kinds of statistical GPGPU experiments, we have observed the similar gamma-distribution-shaped distributions. Sometimes approximation by using $L_{Y}^{\prime}$ happens to give a quite close estimate to the target, and some other times it furnishes just a rough sketch of the actual observations as seen in the example in Section $5 .$.

For most readers, the important thing may not be just how accurate the approximation is, but how instrumental the results given here are for designing statistical GPGPU algorithms. From this viewpoint, we might have already confirmed the following two assertions:

1. Statistical GPGPU could be efficient by employing properly designed algorithms, at least for purposes of a particular kind.

2. Allocation size $k$ to each thread should be large enough with due consideration of resource allotment.

For designing practical GPU algorithms of higher performance, we need additional wellestablished techniques, of course, including memory resource management and memory latency hiding.

\section{Acknowledgement}

The authors would like to express their sincere gratitude to the anonymous referees for their helpful comments. This work is partly supported by Grant-in-Aid for Scientific Research (C) \#24500350.

\section{REFERENCES}

Advanced Micro Devices (AMD), Inc. (2012), Getting Started with SequenceL — Optimizing Parallel Processing Performance and Coding Efficiency with AMD APUs and Texas Multicore Technologies' SequenceL Auto-parallelizing Programming Solution, AMD White Paper, AMD Incorporated.

Ahrens, J. H. and Dieter, U. (1974), Computer methods for sampling from gamma, beta, Poisson and binomial distributions, Computing, 12, 223-246.

Gumbel, E. J. (1947), The distribution of the range, Annals of Mathematical Statistics, 18(3), 384-412.

Iida, M. and Niki, N. (2013), GPGPU for probabilistic algorithms with heavily repeated identical procedures (in Japanese), Proceedings of the 27th Symposium of Japanese Society of Computational Statistics, 35-36.

Lee, A., Yau, C., Giles, M. B., Doucet, A. and Holmes, C. C. (2010), On the utility of graphics cards to perform massively parallel simulation of advanced Monte Carlo methods, Journal of Computational Graphics and Statistics, 19(4), 769-789.

Niki, N., Nakagawa, S. and Inoue, H. (1990), Cumulants of random sum distributions, Communication in Statistics - Theory and Methods, 19(5), 1857-1861.

nVIDIA Corp. (2009), nVIDIA's Next Generation CUDA: Fermi, White Paper, nVIDIA Corporation.

nVIDIA Corp. (2013), Cuda C Programming Guide, nVIDIA Corporation. 
Shimokawabe, T., Aoki, T., Ishida, J., Kawano, K. and Muroi, C. (2011), 145 TFlops performance on 3990 GPUs of TSUBAME 2.0 supercomputer for an operational weather prediction, Procedia Computer Science, 4, 1535-1544.

Wolfram Research Inc. (2013), CUDALink, http://reference.wolfram.com/mathematica/CUDALink/guide/CUDALink.html (January 16, 2014).

(Received: August 26, 2013, Accepted: October 5, 2014) 
